\title{
Relación enfermera-familia en la unidad de cuidados intensivos. Hacia una comprensión empática
}

\author{
Nurse-Family Relationship in the Intensive Care \\ Unit. Towards an Empathic Understanding \\ Relação enfermeiro-família em unidade \\ de terapia intensiva. Em direção a uma \\ compreensão empática \\ Camilo Duque-Ortiz, Rn, Esp., MSc, PhD ${ }^{1 *}$ \\ María Mercedes Arias-Valencia, RN, PhD²
}

Recibido: 2 de junio de $2020 \cdot$ Aceptado: 2 de diciembre de 2020

Doi: https://doi.org/10.12804/revistas.urosario.edu.co/revsalud/a.10059

Para citar este artículo: Duque Ortiz C, Arias-Valencia MM. Relación enfermera-familia en la unidad de cuidados intensivos: hacia una comprensión empática. Rev Cienc Salud. 2021;19(1):1-20. https://doi.org/10.12804/revistas.urosario.edu.co/revsalud/a.10059

\section{Resumen}

Introducción: desde la perspectiva de humanizar el cuidado en la unidad de cuidados intensivos, se ha evidenciado la importancia de prestar más atención a los familiares del paciente e incluirlos en las dinámicas de la unidad. El objetivo del estudio fue comprender la relación entre el profesional de enfermería de la unidad de cuidados intensivos adultos y los familiares, desde la perspectiva de estos últimos y elaborar un planteamiento teórico que describa su proceso de construcción. Materiales y métodos: el estudio se desarrolló bajo el enfoque cualitativo de investigación y la tradición de la teoría fundamentada. Se realizaron 26 entrevistas a familiares de pacientes hospitalizados en diferentes unidades de cuidados intensivos y 100 horas de observación participante entre julio del 2017 y julio de 2019. El análisis de la información se hizo según la propuesta de Strauss y Corbin para la teoría fundamentada. Resultados: se identificó una categoría central (la relación enfermera-familia) hacia una comprensión empática, con cuatro subcategorías: formas de relación; el ideal profesional, la comprensión empática; la construcción de una relación profesional a partir de la interacción, la comunicación y la información, y las barreras en la relación enfermería-familia. Conclusiones: la relación enfermera-familia se fundamenta en la comprensión empática y tiene tres pilares centrales: la interacción, la comunicación

1 Universidad Pontificia Bolivariana (Colombia).

2 Grupo de Investigación Políticas y Servicios de Salud, Universidad de Antioquia (Colombia).

Camilo Duque-Ortiz: orciD https://orcid.org/0000-0003-3106-0471

María Mercedes Arias-Valencia: oRCID https://orcid.org/0000-0003-3876-5976

*Autor de correspondencia: camilo.duque@upb.edu.co 
y la información, ya que propician la comprensión y favorecen la creación de relaciones empáticas y de confianza, lo cual contribuye a la humanización del cuidado profesional.

Palabras clave: relaciones profesional-familia; enfermeros; unidades de cuidados intensivos; relaciones interpersonales; comprensión; empatía.

\section{Abstract}

Introduction: From the perspective of humanizing care in the Intensive Care Unit, the importance of paying more attention to the patient's family members and include them in the dynamics of the unit has become evident. The objective of the study was to understand the relationship between the nursing professional of the Adult Intensive Care Unit and the relatives, from the perspective of the latter and to elaborate a theoretical approach that describes their construction process. Materials and methods: The study was developed under the qualitative approach of research and the tradition of grounded theory. 26 interviews were conducted with relatives of patients hospitalized in the Intensive Care Unit and 100 hours of participant observation between July 2017 and July 2019. The analysis of the information was made based on the proposal of Strauss and Corbin for the grounded theory. Results: A central category was identified, the nurse-family relationship, towards empathic understanding, with four subcategories: relationship forms, the professional ideal, empathic understanding, the construction of a professional relationship based on interaction, communication and information; and the barriers in the nursing-family relationship. Conclusions: The nurse-family relationship is based on empathic understanding and has three central pillars, interaction, communication and information, since they promote understanding and favor the creation of empathetic and trusting relationships, which contributes to the humanization of the professional care.

Keywords: Professional-family relations; nurses; intensive care units; interpersonal relations; familyrelativies; comprehension; empathy.

\section{Resumo}

Introdução: na perspectiva da humanização do cuidado em Unidade de Terapia Intensiva, fica evidente a importância de se dar mais atenção aos familiares do paciente e incluí-los na dinâmica da unidade. $O$ objetivo do estudo foi compreender a relação entre o profissional de enfermagem de unidade de terapia intensiva de adultos e os familiares, na perspectiva destes últimos, e desenvolver uma abordagem teórica que descreva seu processo de construção. Materiais e métodos: o estudo foi desenvolvido sob a abordagem de pesquisa qualitativa e a tradição da teoria fundamentada. Foram realizadas 26 entrevistas com familiares de pacientes internados em unidades de terapia intensiva e 100 horas de observação participante entre julho de 2017 e julho de 2019. A análise das informações foi feita com base na proposta de Strauss e Corbin para a teoria fundamentada. Resultados: identificou-se uma categoria central, a relação enfermeiro-família, no sentido de uma compreensão empática, com quatro subcategorias: formas de relacionamento, o ideal profissional-compreensão empática, a construção de uma relação profissional a partir da interação, a comunicação e informação; e barreiras na relação enfermeiro-família. Conclusões: A relação enfermeiro-família é baseada na compreensão empática e possui três pilares centrais, a interação, a comunicação e a informação, uma vez que promovem a compreensão e favorecem a criação de relações empáticas e de confiança, o que contribui para a humanização do cuidado profissional.

Palavras-chave: relações profissional-família; enfermeiros; unidades de terapia intensiva; relações interpessoais; compreensão; empatia. 


\section{Introducción}

T a relación enfermera-paciente, según Meleis, es la esencia y dominio del cuidado, así como Lun foco central y unificador de la disciplina de enfermería (1). En este sentido, se considera que la enfermería es una relación humana donde se da un proceso interpersonal y terapéutico que favorece la comprensión del significado de la experiencia del paciente (2). De igual forma, se concibe que para brindar un cuidado artístico que responda de manera oportuna a las necesidades del pacientes, es necesario entender los significados que surgen de las relaciones o encuentros con el paciente (3).

El estudio de las relaciones en enfermería se aborda, principalmente, desde la concepción del ser humano como aquel que sufre de una enfermedad o que tiene necesidades de salud. Sin embargo, se ha profundizado poco en las relaciones que establece la enfermera con los familiares del paciente y en contextos específicos y particulares como la unidad de cuidados intensivos (UcI) adultos. En dicha unidad se presentan barreras físicas, normativas, organizativas y humanas que hacen que la interacción y comunicación entre la enfermera y los familiares generen condiciones particulares en el momento de establecer la relación (4,5).

Según Verónica Rojas, el interés por la humanización de los servicios de salud ha llevado a incluir, desde el 2001, el concepto de atención centrada en el paciente y la familia, que busca orientar la atención sanitaria hacia la autonomía y respeto por el paciente y su familia y hacia el diseño de planes de cuidado en los que se integren los valores personales, los patrones de vida y las creencias del paciente y la familia en las terapias establecidas $(6,7)$. Al respecto, Mejías plantea que la familia tiene un papel vital en la vida del paciente, ya que representa parte de su contexto y tiene las importantes funciones de proporcionar recursos físicos y emocionales para mantener la salud de cada uno de sus miembros y de establecer un sistema de apoyo en épocas de crisis, como cuando algún miembro sufre una enfermedad (8).

En la uci, los familiares sufren por la condición de gravedad de su pariente enfermo (9). Dicho sufrimiento se ve agravado por las características de la ucr: unidades cerradas y de horarios restringidos de visitas para ver a los pacientes, lo cual puede llevar a que la familia no se tenga en cuenta en los planes de cuidado y a que no se establezca una relación enfermera-familia (10). Así mismo, la condición de los pacientes que ingresan a la uci, generalmente, se asocia con la muerte, lo que lleva a los familiares a buscar apoyo para afrontar esta situación. Frente a esto, se considera que la enfermera se encuentra en una posición estratégica y, por ende, es a ella a quien acuden los familiares para buscar ayuda $(9,11,12)$.

Para la enfermera, las relaciones que establece con los familiares son necesarias para involucrarlas en la ucr y así brindarles un cuidado directo o indirecto $(8,9,13,14)$. Sin embargo, a pesar de que se han demostrado los beneficios de vincular a la familia al cuidado de los pacientes y, a la vez, considerarlos sujetos de cuidado, en las uci se sigue actuando como si 
fueran unidades cerradas donde persisten problemas de comunicación y de interacción entre las familias y el personal sanitario $(4,5,10)$.

De acuerdo con lo expuesto, el objetivo general fue comprender la relación entre el profesional de enfermería de la uci y los familiares, desde la perspectiva de estos últimos y elaborar un planteamiento teórico que describa su proceso de construcción.

\section{Materiales y métodos}

$E^{1}$ estudio se desarrolló bajo el enfoque cualitativo de investigación y a través del método de la teoría fundamentada, desde la perspectiva de Strauss y Corbin $(15,16)$. Este último se consideró un método favorable para hacer una descripción teórica de la relación entre la enfermera y la familia, al considerar dicho fenómeno de estudio un proceso de naturaleza humana y social.

El estudio contó con participantes de tres clínicas de la ciudad de Manizales y doce de la ciudad de Medellín (Colombia). En esta última se realizó observación participante en las ucI de dos clínicas privadas. Dos instituciones establecían seis horas continuas al día para que los familiares ingresaran a visitar a su paciente. Las instituciones restantes solo disponían de dos horas al día, repartidas en dos jornadas de una hora, para el ingreso de los familiares. Todas las instituciones permitían la presencia de tres familiares en la sala de espera durante el periodo de visitas; sin embargo, solo uno de ellos podía ingresar al interior de la unidad.

La información se recolectó por medio de entrevistas semiestructuradas, las cuales se complementaron con observación participante. Estas se desarrollaron a través de un trabajo de campo de 24 meses, comprendidos entre julio del 2017 y julio del 2019.

En los dos primeros meses del trabajo de campo se realizó un estudio exploratorio con el propósito de preparar a los investigadores y de reconocer aspectos que favorecieran el abordaje de los participantes en las entrevistas y la inmersión en el campo (17). En dicho estudio se llevó a cabo una observación inicial y entrevistas a una trabajadora social con experiencia y formación académica en el manejo de familiares en ucı, a una enfermera investigadora con experiencia en el desarrollo de investigaciones sobre familia en ucı y a dos familiares que vivieron la experiencia de estar en la uci acompañando a un pariente enfermo. En la tabla 1 se describen los aspectos que se reconocieron a través del estudio exploratorio. 
Tabla 1. Aspectos procedimentales del estudio exploratorio, entrevistas, observación participante y análisis

\begin{tabular}{|c|c|c|}
\hline Técnica & Criterio & Descripción \\
\hline \multirow[b]{2}{*}{ Estudio exploratorio } & Observación inicial & $\begin{array}{l}\text { Se reconocieron los horarios de visita, las dinámicas ge- } \\
\text { nerales de los familiares, las posibles barreras físicas y } \\
\text { normativas de la institución y la unidad y distribución } \\
\text { espacial de la uci. }\end{array}$ \\
\hline & $\begin{array}{l}\text { Entrevistas (experto, públi- } \\
\text { co relacionado, investiga- } \\
\text { dor) }\end{array}$ & $\begin{array}{l}\text { Formas como se podía hacer el abordaje y seguimiento } \\
\text { de los familiares, elementos que fueran importantes pa- } \\
\text { ra la recolección de información; claridad y compren- } \\
\text { sión de la guía de preguntas y de aspectos para tener en } \\
\text { cuenta en el abordaje de los participantes. }\end{array}$ \\
\hline \multirow{3}{*}{$\begin{array}{l}\text { Entrevistas } \\
\text { semiestructuradas }\end{array}$} & $\begin{array}{l}\text { Búsqueda y selección de los } \\
\text { participantes }\end{array}$ & $\begin{array}{l}\text { Muestreo por bola de nieve, por criterio u oportunístico } \\
\text { y a conveniencia. } \\
\text { Guiado por muestreo teórico. }\end{array}$ \\
\hline & Criterios de inclusión & $\begin{array}{l}\text { Personas que por parentesco o afiliación se reco- } \\
\text { nocieran como familiares de la persona que estuvo } \\
\text { hospitalizada en la uci. } \\
\text { - Familiares con el rol de representante del paciente } \\
\text { y la familia ante la unidad. } \\
\text { - Familiares que hubieran acompañado al paciente } \\
\text { durante su estancia en la uci. }\end{array}$ \\
\hline & Criterios de exclusión & $\begin{array}{l}\text { Familiares de pacientes: } \\
\text { - Con proceso de reanimación. } \\
\text { - Ingresados en las últimas } 24 \text { horas. } \\
\text { - Limitación de esfuerzo terapéutico. } \\
\text { - En una situación muy crítica y en alto riesgo de morir. }\end{array}$ \\
\hline $\begin{array}{l}\text { Observación } \\
\text { participante }\end{array}$ & Espacios observados & $\begin{array}{l}\text { El interior de la unidad desde la estación de enfermería } \\
\text { y otros puntos que permitieron reconocer las dinámi- } \\
\text { cas internas, la sala de espera, la sala de descanso del } \\
\text { personal y el espacio entre la sala de espera y la unidad, } \\
\text { que corresponde al puesto del vigilante. }\end{array}$ \\
\hline \multirow{7}{*}{ Análisis } & Método & $\begin{array}{l}\text { Pasos según la teoría fundamentada, desde la perspec- } \\
\text { tiva de Strauss y Corbin: microanálisis, codificación } \\
\text { abierta, axial y selectiva. }\end{array}$ \\
\hline & \multirow{3}{*}{ Procedimiento } & Simultáneo a la recolección de información. \\
\hline & & Manual, con apoyo del software Microsoft Excel®. \\
\hline & & Apoyo con diagramas y memos. \\
\hline & \multirow{3}{*}{ Procesamiento de datos } & Elaboración de tarjetas de análisis. \\
\hline & & Obtención de 4379 códigos. \\
\hline & & $\begin{array}{l}\text { Construcción de la estructura de análisis jerárquica con } \\
\text { categorías-subcategorías, propiedades y dimensiones. }\end{array}$ \\
\hline
\end{tabular}

Para las entrevistas, los criterios de inclusión fueron: las personas que por parentesco o afiliación se reconocieran como familiares del paciente, los familiares con rol de representante del paciente ante la unidad y los familiares que acompañaron al paciente en la uci. Se excluyeron del estudio los familiares de pacientes con limitación de esfuerzo terapéutico, en proceso de reanimación, que hubieran ingresado en las últimas 24 horas o que, según el 
criterio del intensivista, se encontrará en una situación muy crítica y de alto riesgo de morir. Esto, teniendo en cuenta que los familiares de dichos pacientes podían tener mayor riesgo de desarrollar una crisis de ansiedad.

Los participantes se buscaron utilizando las técnicas de muestreo: a conveniencia, por criterio u oportunístico y por bola de nieve (18). Para esto, a través de familiares, colegas y amigos, se exploró y se estableció contacto con las personas que cumplieran con los criterios de inclusión y que estuvieran interesados en participar del estudio. La selección de los participantes se orientó por muestreo teórico y el total se determinó por la saturación teórica de las categorías (19). En total fueron 28 entrevistas, de las cuales 26 correspondieron a familiares de pacientes en ucı y 2 a la experta y a la enfermera investigadora, ambas del estudio exploratorio.

Todas las personas invitadas aceptaron participar en el estudio. El investigador principal fue el entrevistador, quien solicitó la firma del consentimiento informado y quien se reunió con ellas en un lugar y a una hora concertada con cada uno de los familiares. Las entrevistas tuvieron una duración aproximada de entre 30 y 90 minutos y se orientaron con una guía de preguntas abiertas y descriptivas con las que se buscó que los familiares describieran su estancia en la ucr; la manera como se relacionaron, interactuaron y comunicaron con las enfermeras, y los acuerdos y consensos a los que llegaron.

Las entrevistas se grabaron y transcribieron, y se complementaron con 100 horas de observación participante, realizada por el investigador principal, con dos propósitos: triangular la información recolectada en las entrevistas y reconocer el entorno en el que se desarrolló dicha relación. La observación se hizo tanto durante los horarios de visita como por fuera de estos, al igual que en diversos días de la semana. De igual forma, en diferentes espacios, como se describe en la tabla 1.

Toda la observación se registró en un diario de campo, además de comentarios reflexivos del investigador. Dicha información se transcribió en un procesador de texto, se almacenó y se codificó.

El proceso de análisis se basó en los pasos de la teoría fundamentada propuestos por Strauss y Corbin; además, se realizó de manera manual con apoyo del software Microsoft Excel® (20). Inició con un microanálisis que consistió en una lectura detallada de las entrevistas y de las observaciones, a fin de identificar unidades de análisis. Estos fragmentos de datos se transcribieron a una tarjeta electrónica diseñada en Excel ${ }^{\circledR}$ en la que se ubicaron los códigos vivos que se encontraron en los datos o se asignaron códigos sustantivos, como se muestra en la figura 1. En este paso, por medio de descripciones, se seleccionó el código que mejor conceptualizara y representara la unidad de análisis. Se construyeron 4379 tarjetas, las cuales equivalieron al total de códigos obtenidos. 


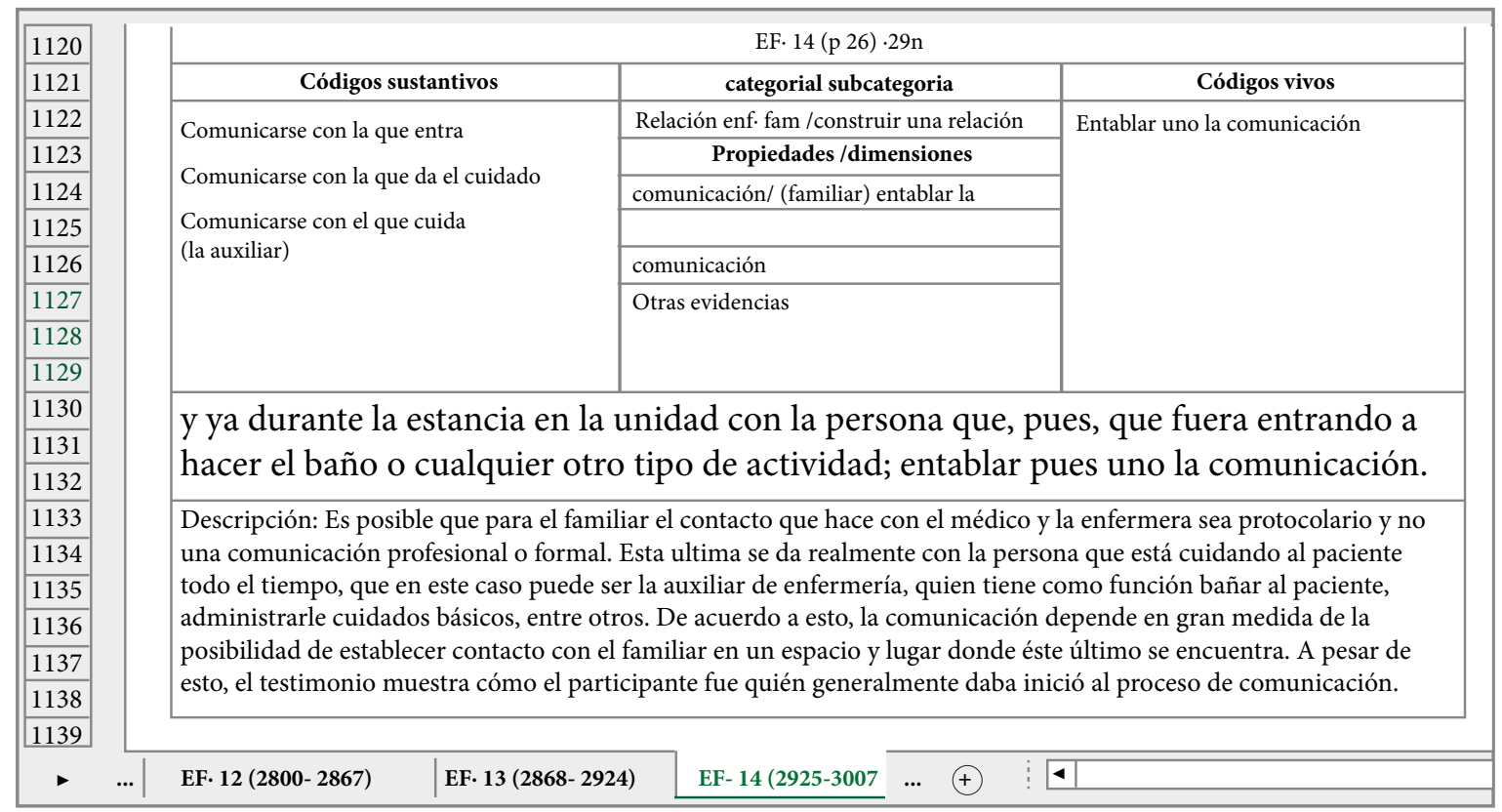

Figura 1. Tarjeta de análisis

Para desarrollar la codificación abierta y axial, se imprimieron las tarjetas electrónicas, con el fin de agruparlas según sus semejanzas y, así, hacer la identificación inicial de categorías, subcategorías, propiedades y dimensiones. Simultáneamente, dichas agrupaciones se sistematizaron en una hoja en Excel®, lo que permitió la organización de los códigos en una estructura categorial, como se muestra en la figura 2. Este proceso se apoyó con la vinculación teórica, lo cual favoreció la interpretación de los datos, el establecimiento de relaciones entre los niveles categoriales y la identificación de la categoría central, la relación enfermera-familia, hacia una comprensión empática. Adicional a lo mencionado, durante el proceso de análisis se utilizaron diagramas, memos analíticos y memos teóricos. 




Figura 2. Estructura categorial del análisis

El análisis se realizó paralelamente con la recolección de información. Para esto se programaron periodos de recolección de datos, seguidos de periodos de análisis, con el fin de favorecer la emergencia de las categorías y el muestreo teórico (19,21). En el desarrollo de la investigación se tuvieron en cuenta los criterios de rigor de credibilidad, auditabilidad y transferibilidad que se describen en la tabla 2 (22).

Tabla 2. Criterios de rigor

Criterio

Realización de ejercicios de reflexividad constantes. Investigador a cargo de la recolección de información, transcripción y análisis. Registro de la información en dispositivos de audio y diarios de campo.

Credibilidad Triangulación de técnicas de recolección de información con la entrevista y la observación participante.

Búsqueda de casos negativos con cada categoría identificada.

Devolución de resultados a las instituciones y participantes.

Auditabilidad La investigación fue compartida con comunidades académicas y pares evaluadores.

Descripción detallada del contexto y de las condiciones de los participantes, identificando

Transferibilidad la aplicabilidad de resultados en espacios y situaciones donde los familiares se ven enfrentados a situaciones similares a las que viven en la uci. 
El estudio se presentó a dos comités de Ética de Investigación de dos instituciones universitarias de la ciudad de Medellín (Colombia), que concedieron su aval mediante las actas 08 de junio de 2017 y cEI-FE 2017-14. Se realizó un protocolo de primeros auxilios psicológicos, el cual fue revisado y ajustado por un profesional en psicología. Lo anterior, considerando que los participantes podían sufrir una descompensación emocional. Sin embargo, no fue necesaria su aplicación, debido a que no hubo alteraciones psicológicas. Incluso, algunos familiares reconocieron las entrevistas como una experiencia liberadora.

Para garantizar la confidencialidad de los participantes, la información recolectada se almacenó en el computador personal del investigador principal y solo el equipo de investigación accedió a ella. Cuando se mencionaron personas, lugares o instituciones, se asignaron seudónimos para su referencia.

\section{Resultados}

\section{Perfil sociodemográfico de los participantes}

En la tabla 3 se describen algunas características sociodemográficas de los 26 familiares entrevistados. De estos, 2 fueron hombres y 24 fueron mujeres, y el promedio de edad fue de 39 años. El parentesco más común entre los familiares entrevistados con relación al paciente fue el de hijos. El $61.5 \%$ de los participantes convivía con el paciente en el momento de ser hospitalizados, el $23.1 \%$ cumplía con el rol de cuidadores antes de llegar a la uci y el 57.7\%, durante la hospitalización del paciente en la ucı, fue responsable de representar a la familia ante la unidad.

Tabla 3. Características de los participantes

\begin{tabular}{lllll}
\hline Sexo & Tipo de familiar/entrevistado & Edad & Ciudad & \multicolumn{1}{c}{ Profesión } \\
\hline Mujer & Trabajadora social & 42 & Medellín & Trabajadora social \\
Mujer & Enfermera investigadora & 36 & Medellín & Enfermera \\
Mujer & Hermana & 41 & Medellín & Secretaria \\
Mujer & Sobrina & 25 & Medellín & Enfermera \\
Mujer & Hija & 28 & Medellín & Enfermera \\
Mujer & Hija & 34 & Manizales & Enfermera \\
Mujer & Hija & 57 & Medellín & Enfermera \\
Mujer & Esposa & 66 & Medellín & Administradora centro educativo \\
Mujer & Hermana & 49 & Medellín & Asistente de fiscal \\
\hline
\end{tabular}

Continúa 


\begin{tabular}{lllll}
\hline Sexo & Tipo de familiar/entrevistado & Edad & Ciudad & \multicolumn{1}{c}{ Profesión } \\
\hline Mujer & Esposa & 47 & Medellín & Auxiliar contable \\
Mujer & Hija & 24 & Medellín & Estudiante universitaria \\
Mujer & Hija & 53 & Medellín & Auxiliar de odontología \\
Mujer & Nieta & 25 & Medellín & Enfermera \\
& & & & Ingeniero mecánico-docente \\
Mujer & Hermano & 42 & Medellín & universitario \\
Hombre & Hijo & 56 & Manizales & Comerciante \\
Mujer & Esposa & 43 & Manizales & Publicista \\
Mujer & Esposa & 52 & Medellín & Ama de casa \\
Mujer & Hija & 23 & Medellín & Terapeuta respiratoria \\
Mujer & Hija & 46 & Medellín & Tecnóloga hemodinámica \\
Mujer & Prima hermana & 33 & Manizales & Contadora pública \\
Mujer & Hija & 33 & Medellín & Auxiliar de enfermería \\
Hombre & Suegra & 63 & Manizales & Pensionado \\
Mujer & Nieta & Manizales & Enfermera \\
Mujer & Nieta & 33 & Manizales & Estudiante odontología \\
Mujer & Hija & 19 & Manizales & Auxiliar de enfermería \\
Mujer & Nieta & 35 & Manizales & Enfermera \\
Mujer & Nieta & 27 & Medellín & Estudiante enfermería \\
Mujer & Hija & 30 & Estudiante enfermería \\
\hline & & 26 & \\
\hline
\end{tabular}

\section{Análisis cualitativo mediante teoría fundamentada}

El análisis de los datos permitió identificar una categoría central: la relación enfermerafamilia, hacia una comprensión empática, con cuatro subcategorías: 1) formas de relación; 2) el ideal profesional, la comprensión empática; 3) la construcción de una relación profesional a partir de la interacción, la comunicación y la información, y 4) las barreras en la relación enfermería-familia. A continuación, se describen dichas categorías con los datos que las sustentan. Las entrevistas realizadas a los familiares se representan como EF, mientras que las realizadas a la experta y la enfermera investigadora se representan como EE y EI, respectivamente.

\section{Formas de relación}

Según los datos, se reconoce que los familiares de los pacientes en la uci consideran que la capacidad de relacionarse de la enfermera se da espontáneamente, porque está implícita en su labor, como parte inherente a su naturaleza profesional. Esto lo refleja el testimonio del siguiente familiar que labora como enfermera: “... debe haber alguien que no por compromiso 
sino porque eso está implícito en la labor...” (ЕF06). De acuerdo con esto, los familiares esperan que la relación con la enfermera se dé naturalmente y que esta contribuya a hacer llevadera la estancia en la uci.

Se observó que la relación entre los familiares y las enfermeras adopta diversas formas, como obligatorias, de puente o lazo de unión, de poder o control, débiles o nulas, de amigos y descentrada (ponerse en el lugar del otro).

De las formas identificadas, la descentrada se acerca a una relación profesional ideal, y es la que más desean los familiares, ya que puede traer grandes beneficios para ellos, por ejemplo, el que la enfermera se ponga en su lugar para que los entienda y los trate como personas y no como desconocidos, según lo describe la siguiente enfermera investigadora: “... ellos se sienten reconfortados porque les hablan de que igual... Esto nos pone a nosotros que tenemos que ponernos en el lugar del paciente y de la familia...” (EI04).

Para los familiares es importante que el personal sanitario demuestre que se están poniendo en sus zapatos y que entiendan lo que viven, lo cual pareciera que es más fácil para las enfermeras que para otros profesionales, como lo considera el siguiente familiar: “... uno muchas veces cree que esa parte humana se va perdiendo, pero.... No creas, por ejemplo, había unas que cuando yo me ponía a hablarle... Hasta lloraban...” (EF16).

\section{El ideal profesional: la comprensión empática}

En la relación enfermera-familia, la comprensión empática o empatía cognitiva, en términos de Fernández Pinto et al., surge como el elemento ideal que debería tener la enfermera para relacionarse con la familia (23). En este sentido, los familiares esperan que la enfermera, además de establecer una relación descentrada, muestre a través de sus palabras y actitudes una real comprensión de la situación que vive el familiar.

Para el familiar es importante que la enfermera conozca la situación en la que se encuentran para que las decisiones que tomen no sean arbitrarias, sino individualizadas, como lo describe la siguiente enfermera investigadora: "ellos, en su relación, saben de qué esta muchacha, que es la única familiar de este señor, trabaja y no puede venir en el horario de visita, hay que establecerle este horario, o sea, empieza a conocerse, entonces yo pienso que así es que es la relación con la enfermera” (Еі04).

La integración de los familiares para el establecimiento de una relación profesional se inicia cuando la enfermera mira al familiar, reconoce su presencia, lo llama por su nombre e intercambia información con él, como lo detalla el siguiente testimonio: "que la enfermera vea a la familia, puede que en ese momento el médico también haya dado una información, pero una mirada, un saludo, pues pa con la familia..." (EI04). Para los familiares, el carisma es parte del ideal que favorece el establecimiento de una relación profesional, como lo considera el siguiente familiar: "la enfermera que está en la uci tiene que tener mucho carisma... Son personas que, para estar allá, tienen que ser personas de muy buen, de mucho carisma” 
(EF10). Así, los familiares esperan ser tratados con tacto, sutileza, simpatía y palabras dulces y alentadoras, como se observa en el testimonio del siguiente familiar: “una sonrisa allá, una palabra dulce... Hace la diferencia” (ЕF01).

Los familiares esperan que las enfermeras se conmuevan y compadezcan de ellos para que puedan desahogarse y expresar sus emociones con acciones como el llanto. Así lo menciona el siguiente familiar: "dejaron que llorara, que hicieran el escándalo, todo, todo... Dejaron que ellas ahí se desahogaran cuando el murió...” (Еғ02). De igual forma, se espera que la enfermera les dé seguridad y tranquilidad para dejar a su pariente enfermo en manos de personas desconocidas como el personal de la unidad, que si bien tienen la preparación para atenderlo, no dejan de ser extrañas, como lo narra el siguiente familiar: "me decía... Porque me gusta, pero váyase tranquilita...” (EF-10).

\section{Construir una relación profesional}

Si bien en los ideales humanos y profesionales se considera que la relación hace parte de la naturaleza del ser humano y de la enfermería, esta no se logra de forma natural, sino que se construye. A partir del análisis de los datos, la construcción de la relación enfermera-familia se fundamenta en la interacción y la comunicación, con su componente de información.

En los datos que aportaron los familiares, estos reconocen la interacción como un elemento que favorece el inicio de una relación y el aprendizaje de lo que sucede con el paciente, como lo describe el siguiente familiar: "el contacto allá directo era, inicialmente, con la enfermera jefe o el médico, según el que estuviera disponible... Uno va aprendiendo de las cosas particulares con las cuales uno va teniendo pues como la relación y yo también le pregunto, en general, a todos los médicos... ¿Qué le están aplicando?, ¿qué es lo que tiene?... Pues todo eso, entonces uno va como adquiriendo ese lenguaje con la interacción” (EF14).

Para una trabajadora social, la enfermera tiene la posibilidad de interactuar más con los familiares, respecto a otros profesionales de la unidad, porque hay cotidianidad en los encuentros que tienen, tal como lo relata a continuación: "ellas conversan con la familia, ellos saben en qué están, más que nosotros porque con ellas... Pues... Con las enfermeras hay cotidianidad, con nosotros no" (EE-03).

Al inicio, los familiares perciben la formalidad y la cordialidad de los actores, y dependiendo de los sucesos que la procedan, será la evolución de esta, como lo describe el siguiente familiar: "al principio, empezó como la cosa bien porque decían, pues, que no pasaba como nada; pues, al principio la relación era como muy cordial porque decían... que no, que estaba bien, que lo estaban tratando que no había problema...” (ef14).

La comunicación entre el familiar y la enfermera suele ser asimétrica. En esta comunicación, los interlocutores no se ubican en un mismo nivel; uno tiene ventaja sobre el otro en cuanto a la posición física cómoda que adoptan, el manejo y acceso de la información y la disposición para compartirla. En este tipo de comunicación, los familiares quedan en 
desventaja con relación al personal de la unidad, como lo describen los familiares: "cuando mi papá pasó a hospitalización... o sea, me di cuenta de que... tenía úlceras por presión, que.... tuvo un manejo que ni siquiera me habían dicho cuando estaba en la uci... que le habían hecho... algunos exámenes que ni siquiera me habían comentado” (Еғ18).

La información en la comunicación asimétrica es más restringida, conveniente y condicionada. Fluye en un solo sentido y el protagonismo recae sobre uno de los interlocutores, generalmente, la enfermera, como lo menciona la trabajadora social: "no me dan información... Enfermería no me da información que yo quiero” (ЕЕ03). En este caso, los familiares se ven cohibidos de tomar parte activa de la comunicación, como expresa el siguiente familiar: "uno ahí no puede ser tan radical, sino que uno tiene que digamos... Callarse muchas cosas con las que uno no está de acuerdo...” (EF14).

\section{Barreras en la relación enfermera-familia}

Se identificó la presencia de barreras físicas, administrativas, normativas y humanas que influyen en la manera en que se construye la relación enfermera-familiar. Las tres primeras emergieron al inicio del análisis de datos; sin embargo, a medida en que el estudio avanzaba, fue emergiendo la barrera humana, hasta posicionarse como la principal. Es posible que el personal naturalice estas barreras, es decir, poco las percibe o no se dan cuenta de su existencia: "las personas que trabajan allá no sé si lo dimensionan como tal, entonces ponen a veces muchas barreras...” (EF02).

Las barreras humanas que se identificaron se constituyen como aquellas que dependen de las actitudes y conductas del personal de la unidad, entre ellas la enfermera. Se encontraron las siguientes: desconfianza entre las partes, atención deshumanizada, mal cuidado del paciente y relación dañada.

Hay ocasiones en las cuales los familiares perciben a la enfermera como poco sincera. Ello se da cuando hay una incoherencia entre lo que dicen y las expectativas sobre la evolución y los resultados del paciente, como lo relata el siguiente familiar: "se maneja un poco como de, como de, ¿cómo dice uno cuando las personas son muy queridas, pero son contradictoras con sus actos?; es como que uno las ve como supersalameritas, superlindas y te dicen... 'Sí, tranquila, todo va a estar bien', pero no te llevan más allá de eso. Para mí es como una cara muy diferente de los que uno cree que son, para mí es otra perspectiva diferente...” (EF11).

Para la familia, la uci es un lugar donde las personas que allí se encuentran no parecen humanos o, por lo menos, les falta humanización, como perciben los siguientes familiares: “¿Qué puedo decir yo?... Cero humanidad, cero humanidad...” (ef06). "Eso es totalmente contradictorio a lo que es la humanidad o lo que es el cuidado de la humanidad o esas cosas... No, yo no lo veía” (EF11). En ese sentido, cuando la actuación del personal no parece tener humanidad, no le da valor agregado a sus actos y su labor la hace de manera rutinaria y protocolaria, como lo relata el siguiente familiar: "si no que ellos te entregan, así como si... 
pues, como si... pues, ya la costumbre se les hace... a está bien, no sé qué... no se detienen a decirle" (EF18).

Desde el punto de vista del familiar, ciertas acciones del personal hacen que la relación con la familia se dañe, como expresa el siguiente familiar: "ya de ahí en adelante la relación entre el personal y nosotros, no, pues cero..." (ЕF-06). Esto lleva a que el familiar se aleje y se pierda la posibilidad de interactuar y comunicarse; ello impide que se llegue a una comprensión empática. Esta situación contrasta con el interés de la familia, la cual, por necesidad o por respuesta a sus emociones, buscan acercarse a la enfermera; sin embargo, estas, con sus acciones, intencionales o descuidadas, cierran la relación, como lo describe el siguiente familiar: "Muy poca relación, porque cuando ellas llegaban pues como a revisarla, aplicar medicamentos, así; entonces no teníamos como casi contacto, ni hablar con ellas...” (eғ09). Esto muestra que la relación enfermera-familia es frágil y puede romperse en cualquier momento si no se cultiva y se madura.

\section{Discusión}

$\Gamma^{n}$ los hallazgos se observa que los familiares esperan que se establezca una relación con caron que los familiares consideran que en la uci sus necesidades son secundarias a las de los pacientes, incluso que no llegan a ser atendidas $(24,25)$. En este sentido, Velasco-Bueno et al. reconocieron que las enfermeras están menos interesadas por las inquietudes de las familias y hay una divergencia significativa en la prioridad de algunas de las preocupaciones que muestran los familiares (26). Lo anterior se presta para que predominen otros tipos de relación que no benefician a los familiares.

En este estudio, en la relación enfermera-familia en la uci, la comprensión empática surge como un ideal profesional. Al respecto, Triana Restrepo expone que la comprensión a través de la empatía lleva a sentir el mundo privado del paciente como si fuera el propio, teniendo la claridad de diferenciar ambos (27). Por lo tanto, la comprensión empática favorece el acceso al mundo subjetivo de la otra persona, pero para que esto resulte en algo positivo es necesario que no solo se experimente el marco de referencia del paciente, sino que se le comunique el significado de dicha comprensión (28).

Los resultados de esta investigación concuerdan con lo expuesto por Strachan et al., quienes consideran que para llegar a la comprensión empática es preciso que la enfermera se acerque al familiar y lo conozca, reconociendo sus particularidades, expectativas y emociones, así como las necesidades que surgen durante su estancia en la uci, en especial cuando es la enfermera a quien acuden los familiares cuando desean aclarar sus dudas (29). 
Sin embargo, en el presente estudio se encontró que la relación que establece la enfermera con la familia del paciente se da por el mérito y esfuerzo de esta última. No se logró reconocer el establecimiento de un objetivo común entre ambos; por el contrario, se observan líneas de actuación diferentes, lo que puede afectar la atención y cuidado integral, el acercamiento al mundo del paciente y la familia, la confianza y seguridad de las familias y la adaptación a la situación que viven $(4,29,30)$.

Al respecto, las familias perciben que las enfermeras no comprenden sus reacciones, que son difíciles, pero humanas $(4,30)$. Así, algunas familias consideran que las enfermeras en la uci no muestran empatía (31,32), ya que, en algunas ocasiones, hablan de forma agresiva $(4,33,34)$, no se preocupan por saber si el familiar está triste, muestran poca preocupación por el estado anímico del familiar, no se acercan a tranquilizarlos si los ven angustiados, no conversan con los familiares, no preguntan por los problemas que enfrentan con la situación de su pariente enfermo, no responden de forma cortés, fruncen la frente cuando les preguntan en varias ocasiones, se muestran molestas, frías, mecánicas y les ocultan hechos importantes (30,32). Lo anterior, en coherencia con los hallazgos de la presente investigación, se constituye como una barrera que daña la relación entre la enfermera y la familia y se distancia del ideal profesional: la comprensión empática.

Si bien la comprensión empática es el ideal de la relación enfermera-familia, para llegar a esta es necesario fundamentar la relación en la interacción, la comunicación y la información. La primera favorece el contacto y el reconocimiento del otro, a través de la acción y reacción; la segunda, la secuencia e intercambio de mensajes, y la tercera, el contenido que se transmite. Lo anterior concuerda con propuestas como la surgida en España, denominada Humanizando los cuidados intensivos (35), que busca estimular el acercamiento entre la enfermera y los demás profesionales de la uci con el paciente y la familia por medio de las siguientes líneas estratégicas: abrir las puertas de la ucı, favorecer la presencia y participación de los familiares en los cuidados intensivos y trabajar en aspectos como la comunicación y la prevención, control y manejo del síndrome poscuidados intensivos $(6,36,37)$.

A pesar de que la comprensión empática es un ideal profesional que, sin duda, favorecería la humanización del cuidado en la uci, es una meta y una necesidad apremiante para los familiares, mientras que las enfermeras se limitan a un intercambio mínimo de información, lo cual evidencia un desencuentro de lógicas inconmensurables entre el familiar y la enfermera. En coherencia con los resultados de este estudio, Koukouli et al. mostraron que los familiares buscan formas efectivas de comunicación con el personal de salud, con el propósito de obtener información, ya que el personal no lo suministra (24). En este sentido, Chang et al. identificaron los familiares se acercan al personal en busca de ayuda y, en especial, información sobre el paciente, la cual esperan que sea verdadera, completa y clara (25). Como lo plantean Kuhn y Feyerabend, la inconmensurabilidad de las lógicas puede darse por 
tener dos perspectivas diferentes, pero especialmente por carecer de un lenguaje común que permita un encuentro $(38,39)$.

Bautista-Rodríguez et al. exponen que aceptar a la familia como un eje central de las acciones asistenciales permite humanizar la uci (32). En concordancia con Bernal-Ruiz y Horta-Buitrago, se reconoce la interacción enfermera-familia como la clave para propiciar una atención integral, lo cual evita efectos psicológicos adversos y genera entornos terapéuticos humanizados (40).

En el presente estudio se identificó que la acción de la enfermera hacia la familia es asimétrica, protocolaria, conveniente y utilitarista, casi mecánica, que limita la interacción a un contacto mínimo. Esta manera de acción profesional aparece vacía de sujeto y de humanidad; además, no tiene valor agregado de calidad. Frente a esto, Mitchell y Aitken encontraron que cuando el personal camina en los zapatos de los familiares, podrá aceptar los retos culturales que se le impone, logrando una mayor inclusión de la familia a la ucI (41). Al respecto, SakDankosky et al. encontraron que los familiares esperan que las enfermeras cooperen más y los vean más como socios que como enemigos, de manera que puedan trabajar juntos, tomar decisiones compartidas y, así, ayudar al paciente en su recuperación $(30,42)$.

Se concluye que la relación profesional enfermera-familia se fundamenta en la comprensión empática; sin embargo, hay un claro desencuentro entre dos lógicas inconmensurables que el familiar trata de revertir por todos los medios posibles, haciendo que la construcción de la relación enfermera-familia recaiga sobre el familiar, quien debe movilizarse en medio de condiciones desfavorables.

La relación enfermera-familia tiene tres pilares centrales: la interacción, la comunicación y la información, ya que propician la comprensión y favorecen la creación de relaciones empáticas y de confianza, lo cual contribuye a la humanización del cuidado profesional.

La comprensión empática surge como el elemento central que debería caracterizar la relación enfermera-familia. Así, se espera que la enfermera muestre, a través de lenguaje verbal y corporal, una real comprensión de la situación que vive el familiar. Por lo tanto, se plantea que una relación profesional es una relación fundamentada en la comprensión empática.

Aunque se reconoce la relación como un elemento central del ser humano y de la disciplina de la enfermería, esta no pasó de ser un pronunciamiento en referencia a la humanización del cuidado, pues, en la práctica, no es coherente con la importancia que se le brinda conceptual y teóricamente a dicho concepto y no se observa su aplicación y desarrollo. Una relación que no permita un avance en la formación de sistemas de relación es lo que Luhmann denomina sistemas sociales simples (43).

Si bien la investigación se llevó a cabo en la ciudad de Medellín e incluyó a personas de la región antioqueña, los resultados tienen el alcance para las ucı donde se atiendan pacientes adultos y estos se separen de la familia; incluso se puede inferir que los hallazgos 
sobre la crisis situacional de la familia y el desencuentro de lógicas pueden transferirse a lo que sucede en unidades como cirugía, urgencias, salas de trabajo de parto, entre otras, con características similares a la ucı, por ejemplo: ser cerradas, con horarios restringidos para su ingreso, altamente tecnológicas, separación por tiempos prolongados entre el familiar y el paciente, situación crítica y aguda y con incremento de la posibilidad de muerte del paciente.

Se sugiere que estudios futuros profundicen en aspectos como la simbolización que hacen los familiares de la ucı y sus actores y la manera como esto determina el rol y posición que adoptan cuando se relacionan con las enfermeras. Así mismo, se sugiere el desarrollo de investigaciones orientadas al reconocimiento de las estrategias y la forma como los familiares afrontan la situación que viven cuando ingresan a la uci. Por otro lado, se propone la realización de estudios sobre la inteligencia emocional de las enfermeras y la forma como influye en el desarrollo de una comprensión empática con los familiares del paciente en ucr.

Adicional a lo anterior, se recomienda desarrollar intervenciones de cuidado fundamentadas en la valoración psicosocial e individualiza de los familiares a partir del reconocimiento de necesidades y estados emocionales, la estructura y funcionalidad familiar y las herramientas y mecanismos de afrontamiento con las que cuenta cada familiar. A partir de esto, se propone realizar estudios experimentales y cuasiexperimentales que permitan reconocer las intervenciones más eficaces y efectivas para el cuidado de los familiares y la construcción de relaciones empáticas.

\section{Contribución de los autores}

T as contribuciones de los autores se dieron de la siguiente manera:

L Camilo Duque Ortiz: selección del tema, escritura del proyecto, recolección de datos, análisis de información y escritura del artículo científico.

María Mercedes Arias-Valencia: dirección metodológica del estudio, revisión y escritura del proyecto, análisis e interpretación de información y escritura del artículo científico.

\section{Conflicto de intereses}

Ninguno declarado. 


\section{Referencias}

1. Meleis AI. The discipline of nursing: perspective and domain. En: Theoretical nursing: development and progress. 5. ${ }^{\mathrm{a}}$ ed. Philadelphia: Lippincott Williams and Wilkins; 2012. p. 87-112.

2. Elers-Mastrapa Y, Gilbert-Lamadrid M del P. Relación enfermera-paciente: una perspectiva desde las teorías de las relaciones interpersonales. Rev Cubana Enferm. 2016;32(4):126-36.

3. Escobar-Castellanos B, Sanhueza-Alvarado O. Patrones de conocimiento de Carper y expresión en el cuidado de enfermería: estudio de revisión. Enferm Cuid Humaniz. 2018;7(1):57-72. https://doi.org/10.22235/ech.v7i1.1540

4. Hekmatpou D, Ebrahimi-Fakhar HR. Addressing disruption in family life. J Nurs Res. 2015;23(2):1. https://doi.org/10.1097/jnr.0000000000000069

5. Pérez A, Nicolás A, Goñi R, Regaira E, Margall M, Asiain M. Restricciones físicas en ucr: su utilización y percepción de pacientes y familiares. Enferm Intensiva. 2012;23(2):77-86.

6. Rojas V.Humanización delos cuidados intensivos. Rev Méd Clín Las Condes.2019;30(2):120-5. https://doi.org/10.1016/j.rmclc.2019.03.005

7. Potter PA, Perry AG. Cuidado a las familias. En: Potter PA, Perry A, editores. Fundamentos de enfermería. Barcelona: Elsevier; 2015. p. 111-24.

8. Mejías M. Interacción del personal de enfermería con los familiares del paciente politraumatizado y su relación con el nivel de estrés de los familiares. Rev Electrónica Portales Médicos [internet]. 2010 [citado 2017 sep 30];1-7. Disponible en: http://www.portalesmedicos.com/publicaciones/articles/2339/3/Interaccion-del-personal-de-Enfermeriacon-los-familiares-del-paciente-politraumatizado-y-su-relacion-con-el-nivel-de-estresde-los-familiares

9. Campo Martínez MC, Cotrina Gamboa MJ. Relación de ayuda al familiar del paciente en situación crítica. Enferm Glob. 2011;10(24):103-9.

10. Achury-Beltrán LF. Panorama general de las visitas en las unidades de cuidado intensivo. Investig Enferm Imagen Desarro. 2014;16(1):61-71. https://doi.org/10.11144/Ja- veriana. IE16-1.pgvu\%0A1

11. Achury DM, Achury LF. Participación familiar en el cuidado de pacientes críticos: una propuesta de fundamentación teórica. Investig Enferm Imagen Desarro. 2013;15(2):137-51.

12. Adams JA, Anderson RA, Docherty SL, Tulsky JA, Steinhauser KE, Bailey DE. Nursing strategies to support family members of Icu patients at high risk of dying. Heart Lung. 2014;43(5):406-15. https://doi.org/10.1016/j.hrtlng.2014.02.001

13. Aliberch-Raurell AM, Miquel-Aymar IM. Necesidad de rol en los familiares del paciente en la unidad de cuidados intensivos. Enferm Intensiva. 2015;26(3):101-11. https://doi. org/10.1016/j.enfi.2015.02.001

14. Buckley P, Andrews T. Intensive care nurses' knowledge of critical care family needs. Intensive Crit Care Nurs. 2011;27(5):263-72. https://doi.org/ 10.1016/j.iccn.2011.07.001 
15. Morse J, Anne P. Principles of data analysis. En: Nursing research: the application of qualitative approaches. 2. ${ }^{a}$ ed. Thosuand Oaks: Springer Science Business Media; 1996. p. 1-18.

16. Delgado-Arias C. La teoría fundamentada: la toma de decisión entre perspectivas. En: La teoría fundamentada: decisión entre perspectivas. Bloomington: AuthorHouse; 2012. p. 33-58.

17. Quivy R, Carpenhoudt L Van. La exploración. En: Manual de investigación en ciencias sociales. México: Limusa; 2005. p. 43-83.

18. Patton MQ. Sampling strategies. En: Qualitative research and evaluation methods. 3. ${ }^{\mathrm{a}}$ ed. Thosuand Oaks: Sage; 2002. p. 243-51.

19. Strauss A, Corbin J. El muestreo teórico. En: Bases de la investigación cualitativa: técnicas y procedimientos para desarrollar la teoría fundamentada. Medellín: Editorial Universidad de Antioquia; 2002. p. 219-35.

20. Strauss A, Corbin J. Bases de la investigación cualitativa: técnicas y procedimientos para desarrollar la teoría fundamentada. Medellín: Editorial Universidad de Antioquia; 2002. 1-335 p.

21. Pedraz A, Zarco J, Ramasco M, Palmar AM. El análisis en la investigación cualitativa. En: Maciá L, editor. Investigación cualitativa. Barcelona: Elsevier; 2014. p. 97-109.

22. Arias MM, Giraldo CV. El rigor científico en la investigación cualitativa. Investig Educ Enferm. 2011;29(3):500-14.

23. Fernández-Pinto I, López-Pérez B, Márquez M. Empatía: medidas, teorías y aplicaciones en revisión. An Psicol. 2008;24(2):284-98.

24. Koukouli S, Lambraki M, Sigala E, Alevizaki A, Stavropoulou A. The experience of Greek families of critically ill patients: exploring their needs and coping strategies. Intensive Crit Care Nurs. 2018;45:44-51. https://doi.org/10.1016/j.iccn.2017.12.001

25. Chang PY, Wang HP, Chang TH, Yu JM, Lee SY. Stress, stress-related symptoms and social support among Taiwanese primary family caregivers in intensive care units. Intensive Crit Care Nurs. 2018;49:37-43. https://doi.org/10.1016/j.iccn.2018.05.002

26. Velasco-Bueno JM, Alonso-Ovies A, Heras La Calle G, Zaforteza-Lallemand C. Main information requests of family members of patients in intensive care units. Med Intensiva. 2018;42(6):337-45. https://doi.org/10.1016/j.medine.2018.05.004

27. Triana Restrepo MC. Efecto del vínculo empático enfermera-paciente sobre el nivel de ansiedad del paciente adulto en la unidad de cuidado intensivo [tesis de doctorado en internet]. Bogotá: Universidad Nacional de Colombia; 2016 [citado 2018 nov 4]. Disponible en: http://www.bdigital.unal.edu.co/55992/1/51798649.2017.pdf

28. Olivera J, Braun M, Roussos A. Instrumentos para la evaluación de la empatía en psicoterapia. Rev Argent Clín Psicol. 2011;20(2):121-32.

29. Strachan PH, Kryworuchko J, Nouvet E, Downar J, You JJ. Canadian hospital nurses' roles in communication and decision-making about goals of care: an interpretive description of critical incidents. Appl Nurs Res. 2018;40:26-33. https://doi.org/10.1016/j. apnr.2017.12.014 
30. Sak Dankosky N, Andruszkiewicz P, Sherwood PR, Kvist T. Preferences of patients' family regarding family-witnessed cardiopulmonary resuscitation: a qualitative perspective of intensive care patients' family members. Intensive Crit Care Nurs. 2018;1-8. https:// doi.org/10.1016/j.iccn.2018.04.001

31. Kisorio LC, Langley GC. End-of-life care in intensive care unit: family experiences. Intensive Crit Care Nurs. 2016;35:57-65. https://doi.org/10.1016/j.iccn.2016.03.003

32. Bautista-Rodríguez LM, Arias-Velandia MF, Carreño-Leiva ZO. Percepción de los familiares de pacientes críticos hospitalizados respecto a la comunicación y apoyo emocional. Rev Cuid. 2016;7(2):1297-309. https://doi.org/10.15649/cuidarte.v7i2.330 Rev

33. Ayuso-Murillo D, Colomer-Sánchez A, Herrera-Peco I. Habilidades de comunicación en enfermeras de uci y de hospitalización de adultos. Enferm Intensiva. 2017;28(3):105-13. https://doi.org/10.1016/j.enfi.2016.10.006

34. Thermaenius J, Schandl A, Sluys KP. Development and Initial validation of the Swedish Family Satisfaction Intensive Care Questionnaire (SFS-ICQ). Intensive Crit Care Nurs. 2019;50:118-24. https://doi.org/10.1016/j.iccn.2018.05.003

35. Humanizando los Cuidados Intensivos. Manual de buenas prácticas de humanización en unidad de cuidados intensivos. Madrid: Proyecto Hu-cr; 2017 [citado 2018 nov 4]. http:// humanizandoloscuidadosintensivos.com/es/buenas-practicas

36. Kozub E, Scheler S, Necoechea G, O’Byrne N. Improving nurse satisfaction with open visitation in an adult intensive care unit. Crit Care Nurs Q. 2017;40(2):144-54. https://doi. org/10.1097/CNQ.0000000000000151

37. De la Fuente-Martos C, Rojas-Amezcua M, Gómez-Espejo MR, Lara-Aguayo P, MoránFernández E, Aguilar-Alonso E. Implantación de un proyecto de humanización en una unidad de cuidados intensivos. Med Intensiva. 2018;42(2):99-109. https://doi.org/10.1016/j.medin.2017.08.002

38. Kuhn T. La resolución de las revoluciones. En: La estructura de las revoluciones científicas. Ciudad de México: Fondo de Cultura Económica; 1971. p. 224-47.

39. Feyerabend P. Apéndice 5. En: Tratado contra el método. Madrid: Tecnos; 1986. p. 282-9.

40. Bernal-Ruiz D, Horta-Buitrago S. Cuidado de enfermería para la familia del paciente crítico desde la teoría de la comprensión facilitada. Enferm Univ. 2014;11(4):154-63. https://doi.org/10.1016/S1665-7063(14)70928-5

41. Mitchell ML, Aitken LM. Flexible visiting positively impacted on patients, families and staff in an Australian intensive care unit: a before-after mixed method study. Aust Crit Care. 2017;30(2):91-7. https://doi.org/10.1016/j.aucc.2016.01.001

42. De Beer J, Brysiewicz P. The conceptualization of family care during critical illness in KwaZulu-Natal, South Africa. Heal SA Gesondheid. 2017;22:20-7. https://doi.org/10.1016/j. hsag.2016.01.006

43. Luhmann N. Sistema y entorno. En: Sistemas sociales: lineamientos para una teoría general. 2. a ed. Barcelona: Anthropos; 1998. p. 172-98. 